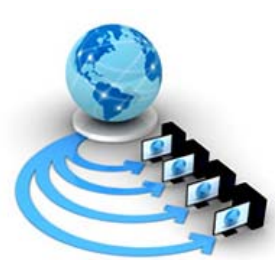

\title{
OPTIMIZED RETINAL BLOOD VESSEL SEGMENTATION TECHNIQUE FOR DETECTION OF DIABETIC RETINOPATHY
}

\author{
Sukhpreet Kaur \\ Research Scholar \\ IKG Punjab Technical University \\ Kapurthala, India
}

\author{
Dr. Kulwinder Singh Mann \\ Professor \\ Guru Nanak Dev Engineering College \\ Ludhiana, India
}

\begin{abstract}
Most of the retinal diseases namely retinopathy, occlusion etc., can be identified through changes exhibited in retinal vasculature of gathered images. Thus, segmentation of retinal blood vessels aids in detecting the alterations and hence the disease. In one data set there are large numbers of features but not all are useful in classification. The useless redundant features reduce the performance of output. In our case we have used only small relevant features rather than using all the features that contain both relevant and irrelevant features. To achieve better results there is need to minimize the number of features along with maximizing the performance of classification. In this paper, to achieve the objective firstly we have extracted the features using the Principle component analysis (PCA) for both series of vessels and non vessel images (normal and abnormal). Then further extracted features are optimized using various optimization methods which actually optimize the selected features of images taken from DRIVE dataset for both vessels and non-vessels to improve the recognition accuracy. Afterwards, the naive bayes classifier has been applied to test the results for both normal and abnormal images dataset. The accuracy of PSO optimization is 0.99537 which is $31.42 \%$ better than LION and $53.09 \%$ better than Firefly. The simulation results show that PSO is better in terms of accuracy, sensitivity, entropy, etc., as compared to other optimization methods for both normal and abnormal set of images.
\end{abstract}

Keywords: Vessel images, Non vessel images, Particle Swarm Optimization (PSO), Firefly, LION, Accuracy, Sensitivity

\section{INTRODUCTION}

The Retinal vessel segmentation can be utilized to diagnosis, screening, treatment, and evaluation of cardiovascular and ophthalmologic diseases like diabetes, hypertension, arteriosclerosis and chorodial neovascularisation. We can utilize attributes of retinal blood vessels like length, width, tortuosity, angles and branching pattern [2]. The retinal vessels automated segmentation is considered as a first step for ophthalmic disorder in computer aided diagnosis system development [3]. The diabetic retinopathy [4], vessel diameter measurement in relation with diagnosis of hypertension [5], computer assisted laser surgery [6] etc are screening programs. They are implemented by analysis and automatic detection of the vasculature [7]. A large number of death and visual loss has been occurred due to spreads of diabetes on retina [8].

The supervised and unsupervised are the two categories on which automatic segmentation method of retinal blood vessels is categorised. The manually labelled samples are used in case of supervised learning for already trained classifier. The vessels and non vessels are the two classes on which samples pixels are classified. They depend on preclassified data that may not be available in real life applications. Mainly in health images, the training used in this method makes it better than unsupervised methods in terms of performance.

The use of retinal vascular tree in biometric identification is unique for every individual data [9.10]. The manual segmentation of retinal blood vessels is very tedious, long task and it also requires training. The retinal blood vessels automatic segmentation is proved to be very challenging due to various structures complication that is influenced by other sources.
There is change in appearance, shape and orientations of vessels that causes difficulties. There are other also factors such as low contrast between the blood vessels and its background; disturbances caused by the presence of noise. The abnormal structures like diseased regions and lesions, exudates, microaneurysms also exist in it. The misinterpretation of background to vessels, missing or overlooking thin vessels also mislead by automatic vessel segmentation algorithms.

In this research, we have used a new approach that exists CLAHE (Contrast limited adaptive histogram equalization), Edge detection for segmentation, PCA is used for feature extraction then classification has been done using NAIVE BIAS. The whole paper is divided into different sections. We have performed some pre-processing steps for both normal and abnormal vessel images. After pre-processing we have applied Firefly, Lion and PSO on it individually. The simulation results are verified using MATLAB.

Section.1: CLAHE (Contrast limited adaptive histogram equalization)

In order to analyze image in a superior way there is need of pre-processing the image. The contrast enhancement is a technique for pre-processing that is classified into local and global. In paper [11], authors have given a comparative review on these contrast enhancement techniques along with their impact on color image segmentation. They have mainly discussed histogram equalization, histogram specification, AHE and BSB-CLAHE four techniques. The results show that utilization of CLAHE based local contrast enhancement gives optimal results as compared to other global enhancement techniques. In paper [12], authors have used the same CLAHE and one other Wiener filter method for enhancing image. The filters are used to remove the noise present in image and to transfer image into suitable dynamic 
range they have used Gamma correction techniques. In last they have used CLAHE to avoid amplification of unwanted noise present in image and its parameters also help to limit the contrast in homogeneous area. In x-ray images and in other images it is critical to perform image enhancement for getting good segmentation. In autonomous disease diagnosis system, subsequent modules are used to increase image sharpness using magnification of the contrast. In this paper [13], authors have analyzed various pre-processing techniques for segmentation of vertebral bone. They have considered histogram equalization (HE), gamma correction (GC) and contrast limited adaptive histogram equalizer (CLAHE) three methods. The experimental results show that most accurate technique is CLAHE as compared to other techniques. The use of CLAHE gives good result that is why in this paper we have used this technique for image enhancement [14]. In first step we have uploaded an image and then pre-processed it using Contrast Limited Adaptive Histogram Equalization (CLAHE) technique.

Section. 2: Edge detection of segmentation and Feature Extraction (PCA) The second section and step is to detect the edges.

In automated retinopathy analysis system an important clinical role is played by blood vessel detection and segmentation technique. There is need of expertise and time to manually segment an image [15]. An image is divided into essential regions using various methods. The local or abrupt changes are considered for segmenting image using edge detector. The retinal analysis will become efficient using computational intelligence. In our paper, we have used MATLAB graphical user interface (GUI) tool to segment a blood vessels and then extracted the features using Principle component analysis (PCA). In paper [16], authors have used DRIVE database and achieves accuracy of $94.61 \%$ with $73.38 \%$ of sensitivity as compared to existing techniques of blood vessel segmentation. The image can be detected using various existing techniques which identify an image and then locate sharp discontinuities in it. The discontinuities are nothing it is just abrupt changes in pixel density that characterized boundaries [17]. In case of noisy images it is difficult to perform edge detection because there are a large frequency content which is available in both edges and noises. There are number of edge detection techniques available such as Sobel, Robert, Canny, Prewitt and Laplacian of Gaussian (LoG).

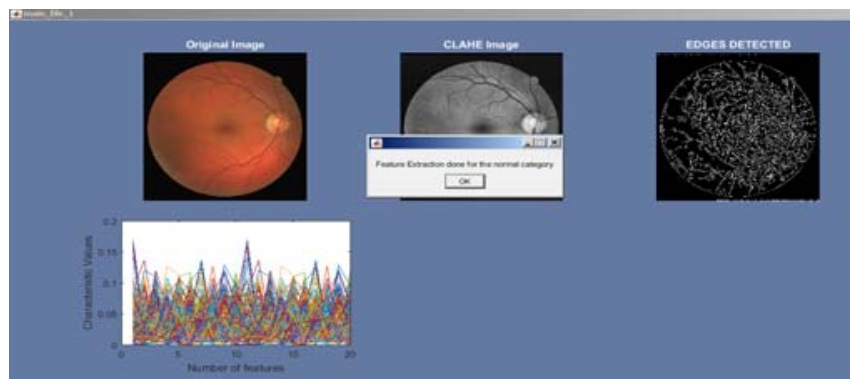

Fig.ure 1: Features extracted for normal image

After segmenting an image using edge detection the next step is feature extraction. The features are extracted using Principle component analysis (PCA) from series of vessels and non vessel images [18]. There is some information which is present in one single image and to extract that we have used feature extraction method. The pattern recognition, feature extractions are the parts of preprocessing steps that helps in extracting and building features which are of foremost importance. In feature extraction we mainly reduce the dimensionality that restricts the resources which is the main set of required data. This reduced and required information will be further manipulated according to our requirement [19]. In our work we want to enhance blood vessels images for this we have used PCA as a feature extractor.

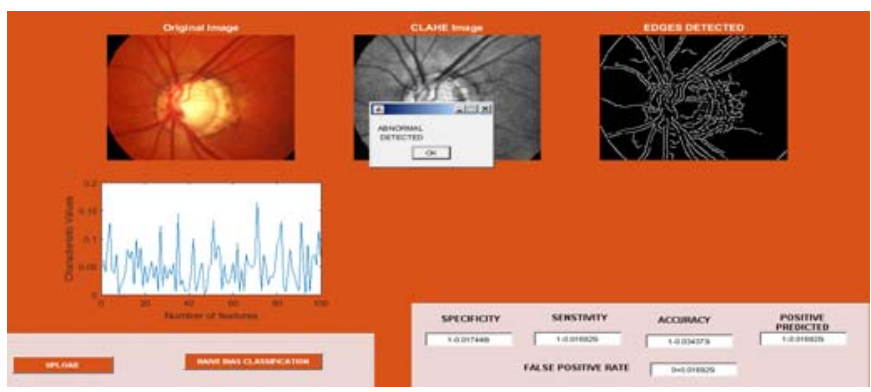

Figure 2: Feature extracted for abnormal image

\section{Section 3: Feature Optimization (Firefly + PSO + LION)}

In second section features has been extracted for both normal and abnormal images using PCA. In this section we will optimize the extracted features using different algorithms. Firstly we have applied particle swarm optimization (PSO) on extracted features. The PSO is proving to be very efficient for feature extraction due to its ability of rarely falling into local optimal. In paper [20], authors have given different pros and cons of PSO algorithms that need to be resolved. PSO is a meta-heuristic nature inspired technique that simulates the bird flocking behaviour. It uses randomly generated population that search for optimal solution by updating velocity and position according to gbest and pbest value. The MATLAB is used for performing simulation results in terms of different parameters [21]. After the feature optimization process, the test images will be classified as normal and abnormal images using classification process using Naive Bayes classifier and performance will be evaluated. The features are optimized and simulation results are given for both normal and abnormal images [22].

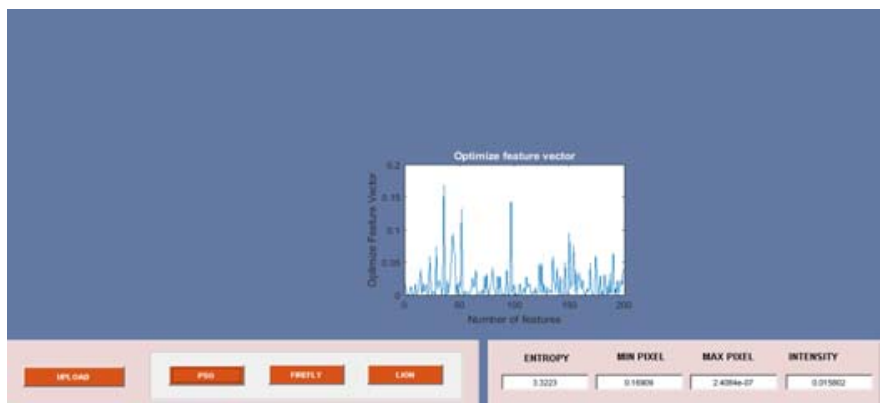

Figure 3: Normal image Feature optimized using PSO 


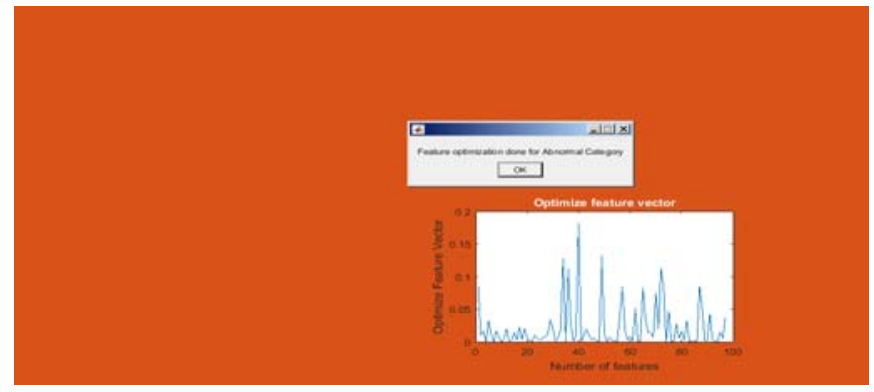

Figure 4: Abnormal image Feature optimized using PSO

Table I: This table shows the simulation results for normal images using PSO, Firefly \& LION:

\begin{tabular}{|l|l|l|l|l|l|}
\hline \multirow{2}{*}{$\begin{array}{l}\text { Algorit } \\
\text { hm }\end{array}$} & \multicolumn{5}{|c|}{ Normal images } \\
\cline { 2 - 6 } & $\begin{array}{l}\text { Sensit } \\
\text { ivity }\end{array}$ & $\begin{array}{l}\text { Specifi } \\
\text { city }\end{array}$ & $\begin{array}{l}\text { Accura } \\
\text { cy }\end{array}$ & $\begin{array}{l}\text { Positive } \\
\text { Predicte } \\
\text { d }\end{array}$ & $\begin{array}{l}\text { False } \\
\text { Positive } \\
\text { Rate }\end{array}$ \\
\hline PSO & $\begin{array}{l}0.9976 \\
6\end{array}$ & 0.99539 & 0.99537 & 0.99539 & $\begin{array}{l}0.00463 \\
04\end{array}$ \\
\hline Firefly & $\begin{array}{l}0.0237 \\
14\end{array}$ & 0.51186 & 0.46443 & 0.04633 & 0.48814 \\
\hline LION & $\begin{array}{l}0.9943 \\
5\end{array}$ & 0.68678 & 0.68112 & 0.76045 & 0.31322 \\
\hline
\end{tabular}

Table II: This table shows the simulation results for Abnormal images using PSO, Firefly \& LION

\begin{tabular}{|l|l|l|l|l|l|}
\hline $\begin{array}{l}\text { Algor } \\
\text { ithm }\end{array}$ & \multicolumn{5}{|c|}{ Abnormal images } \\
\cline { 2 - 6 } & $\begin{array}{l}\text { Sensitivit } \\
\mathbf{y}\end{array}$ & $\begin{array}{l}\text { Specificit } \\
\mathbf{y}\end{array}$ & $\begin{array}{l}\text { Accurac } \\
\mathbf{y}\end{array}$ & $\begin{array}{l}\text { Positive } \\
\text { Predict } \\
\text { ed }\end{array}$ & $\begin{array}{l}\text { False } \\
\text { Positiv } \\
\text { e Rate }\end{array}$ \\
\hline PSO & 0.99448 & 0.70136 & 0.69584 & 0.76906 & $\begin{array}{l}0.2986 \\
4\end{array}$ \\
\hline $\begin{array}{l}\text { Firefl } \\
\text { y }\end{array}$ & 0.023714 & 0.51186 & 0.46443 & 0.04633 & 0.4881 \\
\hline LION & 0.99435 & 0.68678 & 0.68112 & 0.76045 & 0.3132 \\
& & & & & 2 \\
\hline
\end{tabular}

The above shown Table I and Table II gives the results of PSO, Firefly and LION algorithm in terms of Sensitivity, Specificity, Accuracy, Positive and False positive predicted value for both normal and abnormal images.

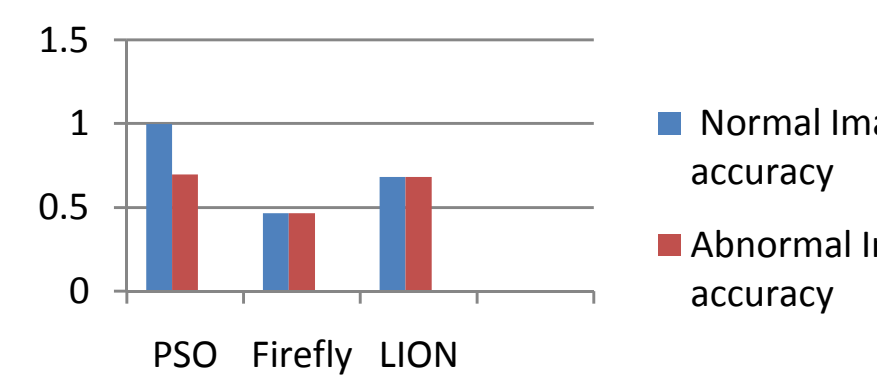

Figure 5: Graph for Table1 and Table2 accuracy value

The above shown fig. 5 is the graph for accuracy value using different optimization algorithms such as PSO, Firefly and LION for both normal and abnormal images.

The firefly will get attracted towards the brighter one if no brighter firefly exist then it will randomly move to get the optimized result [23]. We have used DRIVE data set and normally data sets include number of attributes that are irrelevant and redundant that reduces the accuracy of classification. This algorithm will quickly search for optimal and near optimal feature subset that minimize the used fitness function [24]. The use of firefly for feature optimization does not give an optimized result in terms of different parameters that can be analyzed through simulation results given in tables.

We have analyzed the results for feature optimization using PSO algorithm. Now we will use firefly algorithm for the same [24]. The task of machine learning and data mining performance has been degraded due to noisy and high dimensional data that have large number of features.

The available data is divided into bulk into meaningful, useful groups using clustering. There is no need of any prior information of data and LION optimization is used for clustering.

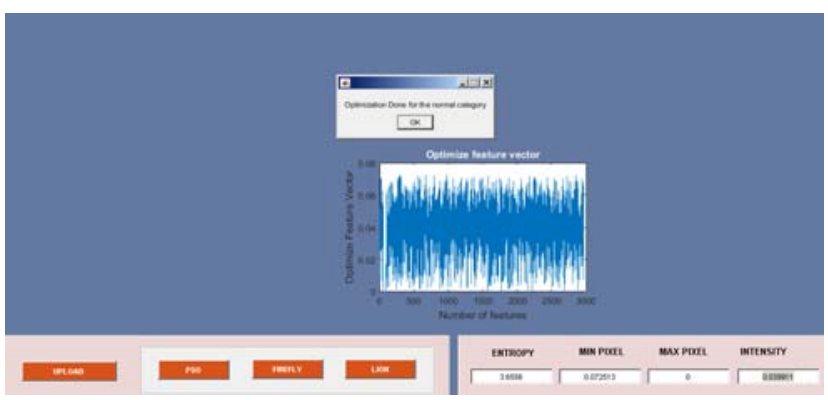

Figure 6: Normal image Feature optimized using LION

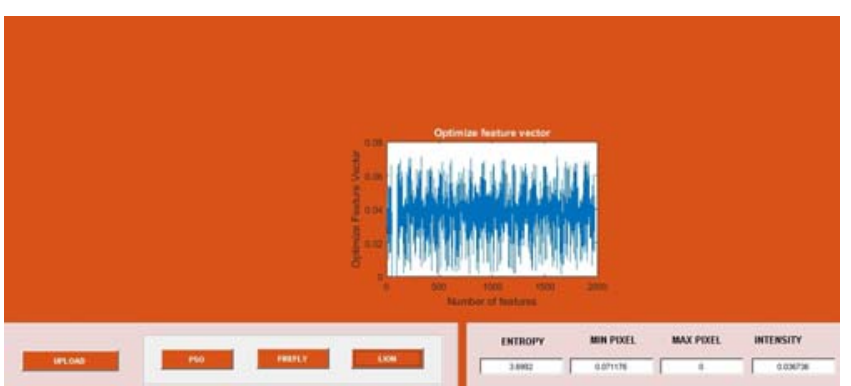

Figure 7: Abnormal image Feature optimized using LION 
After the feature optimization process, the test images will be classified as normal and abnormal images using classification process using Naive Bayes classifier and performance will be evaluated.

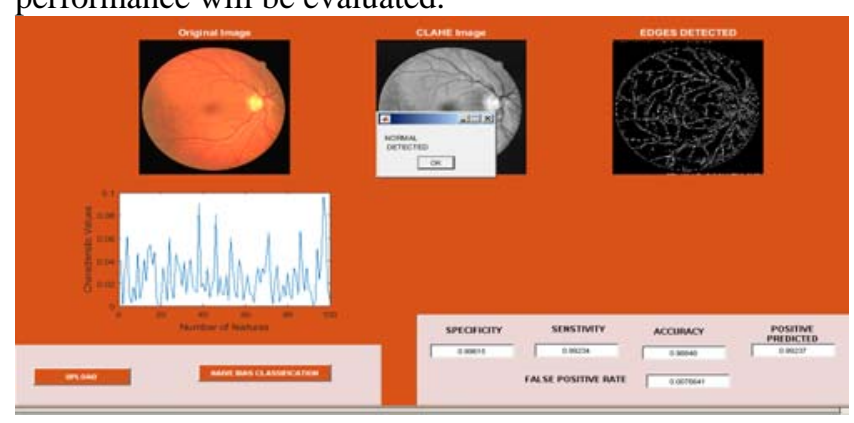

Figure 8: Classification as Normal and Abnormal images using Naïve Bayes classifier

Table III: This table shows the simulation results for normal images using PSO, Firefly \& LION

\begin{tabular}{|c|l|l|l|l|}
\hline $\begin{array}{l}\text { Optimization } \\
\text { Technique }\end{array}$ & \multicolumn{4}{|c|}{ Normal image } \\
\hline & Entropy & $\begin{array}{l}\text { Min } \\
\text { Pixel }\end{array}$ & $\begin{array}{l}\text { Max } \\
\text { Pixel }\end{array}$ & Intensity \\
\hline PSO & 3.3223 & 0.16909 & $\begin{array}{l}2.4084 \mathrm{e}- \\
07\end{array}$ & 0.015802 \\
\hline Firefly & 0.090793 & 99.6888 & 0.12702 & 49.9441 \\
\hline LION & 3.6556 & 0.072513 & 0 & 0.039911 \\
\hline
\end{tabular}

Table IV: This table shows the simulation results for abnormal images using PSO, Firefly \& LION

\begin{tabular}{|c|l|l|l|l|}
\hline $\begin{array}{l}\text { Optimization } \\
\text { Technique }\end{array}$ & \multicolumn{4}{|c|}{ Abnormal image } \\
\hline & Entropy & $\begin{array}{l}\text { Min } \\
\text { Pixel }\end{array}$ & $\begin{array}{l}\text { Max } \\
\text { Pixel }\end{array}$ & Intensity \\
\hline PSO & 3.6803 & 0.4352 & $\begin{array}{l}1.7083 e- \\
05\end{array}$ & 0.029855 \\
\hline Firefly & 0.080793 & 99.1421 & 0.95455 & 50.9886 \\
\hline LION & 3.6992 & 0.071176 & 0 & 0.036736 \\
\hline
\end{tabular}

The above shown Table3 and Table4 gives result of PSO, Firefly and Lion algorithms in terms of Entropy, Min Pixel, Max Pixel and intensity for both normal and abnormal images.

\section{CONCLUSION}

In this paper, traditional feature selection methods and evolutionary algorithms such as PSO, Firefly and LION for feature selection has been investigated. We have used DRIVE dataset and all data sets contain both redundant and useful data. Our aim is to only work on useful data that will give fruitful and accurate results. The use of redundant data will reduce the success rate of getting the output. We have used vessels and non vessels images after uploading and processing the input image. The images will be pre- processed using CLAHE algorithm and edges are detected by performing segmentation. The features of images are extracted using PCA algorithm for both normal and abnormal images. The normal images are those that don't have symptoms of diabetic retinopathy and non vessels or abnormal images are vice a versa. The extracted features are optimized by applying different optimization algorithms. We have used MATLAB and analyzed results in terms of sensitivity, Specificity, Accuracy, Positive and false positive predicted rate. The accuracy of PSO optimization is 0.99537 which is $31.42 \%$ better than LION and $53.09 \%$ better than Firefly. The results are good for normal images but in case of abnormal images the results are not very efficient. To further increase the accuracy for abnormal images a hybrid approach is desirable.

\section{ACKNOWLEDGEMENT}

Authors are highly thankful to the RIC department of IKG Punjab Technical University, Kapurthala, Punjab, India for providing the opportunity to conduct this research work.

\section{REFERENCES}

[1] M.M. Fraz, P. Remagnino, A. Hoppe, B. Uyyanonvara, A.R. Rudnicka, C.G. Owen, S.A. Barman, "Blood vessel segmentation methodologies in retinal images: A survey", Computer methods and programs in biomedicine, vol. 108, pp. 407-433, 2012.

[2] J.J. Kanski, Clinical Ophthalmology, 6th ed., Elsevier Health Sciences, London, UK, 2007.

[3] M.M. Fraz, P. Remagnino, A. Hoppe, B. Uyyanonvara, A.R. Rudnicka, C.G. Owen, S.A. Barman, "Blood vessel segmentation methodologies in retinal imagesa survey”, Comput. Methods Programs Biomed, vol. 1, pp. 407-433, 2012.

[4] T. Teng, M. Lefley, D. Claremont, "Progress towards automated diabetic ocular screening: a review of image analysis and intelligent systems for diabetic retinopathy”, Medical and Biological Engineering and Computing, vol. 40, pp. 2-13, 2002.

[5] C. Heneghan, J. Flynn, M. O’Keefe, M. Cahill, "Characterization of changes in blood vessel width and tortuosity in retinopathy of prematurity using image analysis”, Medical Image Analysis, vol. 6, pp. 407-429, 2002.

[6] J. Lowell, A. Hunter, D. Steel, A. Basu, R. Ryder, R.L. Kennedy, "Measurement of retinal vessel widths from fundus images based on 2-D modelling”, IEEE Transactions on Medical Imaging, vol. 23, pp. 11961204, 2004.

[7] F. Zana, J.C. Klein, “A multimodal registration algorithm of eye fundus images using vessels detection and Hough transform”, IEEE Transactions on Medical Imaging, vol. 18, pp. 419-428, 1999.

[8] K. Verma, P. Deep, A.G. Ramakrishnan, "Detection and classification of diabetic retinopathy using retinal images flight', Annual IEEE India Conference (INDICON), vol. 4, pp. 1-6, 2011.

[9] C. Marino, G. Penedo, M. Penas, J. Carreira, F. Gonzalez, "Personal authentication using digital retinal images”, Pattern Analysis and Applications, vol. 9, pp. 21-33, 2006. 
[10] C. Kose, C. Ikibas, “A personal identification system using retinal vasculature in retinal fundus images", Expert Systems with Applications, vol. 38, pp. 13670-13681, 2011.

[11] Dibya Jyoti Bora, “Importance of Image Enhancement Techniques in Color Image Segmentation: A Comprehensive and Comparative Study”, Indian J.Sci.Res. 15, vol. 4, pp. 115-131, 2017.

[12] Mithilesh Kumar, Ashima Rana, “Image Enhancement using Contrast Limited Adaptive Histogram Equalization and Wiener filter”, International Journal Of Engineering And Computer Science, vol. 5, pp. 16977-16979, 2016.

[13] Bhaswati Das, Deepak Sharma, "Performance Enhancement of Content Based Image Retrieval System Using Contrast Limited Adaptive Histogram Equalization”, Research Inventy: International Journal Of Engineering And Science, vol. 3, pp. 2936, 2013.

[14] Ili Ayuni Mohd Ikhsan, Aini Hussain, Mohd Asyraf Zulkifley, Nooritawati Md. Tahir, Aouache Mustapha, "An Analysis of X-Ray Image Enhancement Methods for Vertebral Bone Segmentation”, 2014 IEEE 10th International Colloquium on Signal Processing \& its Applications (CSPA2014), vol. 6, pp. 208-211, 2014.

[15] Fari Muhammad Abubakar, "Study Of Image Segmentation By Using Edge Detection Techniques”, International Journal of Engineering Research \& Technology (IJERT), vol. 1, pp. 1-5, 2012.

[16] Muthukrishnan.R, M.Radha, "Edge detection techniques for image segmentation”, International Journal of Computer Science \& Information Technology (IJCSIT), vol. 3, pp. 260-267, 2011.

[17] Claudia Kondermann, Daniel Kondermann, Michelle Yan, "Blood Vessel Classification into Arteries and Veins in Retinal Images”, IEEE, pp. 1047-1052, 2007.
[18] Swapnil Saxena1, Vidushi Sharma, "Retinal Blood Vessel Segmentation Using Graphical User Interface (GUI) for Diagnosis of Disease”, International Journal of Innovative Research in Science, Engineering and Technology, vol. 5, pp. 7290-7295, 2016.

[19] Binjie Xiao, "Principal component analysis for feature extraction of image sequence", 2010 International Conference on Computer and Communication Technologies in Agriculture Engineering, vol. 15, pp. 250-253, 2010.

[20] Neha, Jyoti Vashishtha, "Particle Swarm Optimization based Feature Selection”, International Journal of Computer Applications, vol. 146, pp. 1116, 2016.

[21] Bing Xue, Mengjie Zhang, Will N. Browne, "Particle Swarm Optimization for Feature Selection in Classification: A Multi-Objective Approach", IEEE TRANSACTIONS ON CYBERNETICS, vol. 9, pp. 1-12, 2012.

[22] Ayan Seal, Suranjan Ganguly, Debotosh Bhattacharjee, Mita Nasipuri and Consuelo GonzaloMartin, "Feature Selection using Particle Swarm Optimization for Thermal Face Recognition", Springer Applied computation and security systems, vol. 1, pp. 22-35, 2015.

[23] Hema Banati , Monika Bajaj, "Fire Fly Based Feature Selection Approach", IJCSI International Journal of Computer Science Issues, vol. 8, pp. 473480, 2011.

[24] S. Chander, P. Vijaya, P. Dhyani, "Fractional Lion Algorithm - An Optimization Algorithm science for Data Clustering”, Journal of Computer Science, Vol 17, pp 323-340, 2016.

[25] C. Kondermann, D Kondermann, M. Yan, “ Blood Vessel Classification into Arteries and Veins in Retinal Images”, Vol. 8, pp 125-134, 2012. 